\title{
Optical Yarn Hairiness Measurement System
}

\author{
Vitor H. Carvalho, Paulo J. Cardoso, Rosa M. Vasconcelos, Filomena O. Soares and Michael S. \\ Belsley
}

\begin{abstract}
This paper presents a system developed for measuring yarn hairiness using a coherent optical signal processing technique, in steps of $1 \mathrm{~mm}$. The system hardware is divided into two main parts: optical, for establishing an image regarding yarn hairiness and electronic, which converts the optical signal to a proportional voltage signal. Additionally, software using LabVIEW ${ }^{\mathrm{TM}}$ was also designed to acquire the output voltage with a Data Acquisition Board (DAQ) and to perform the corresponding data processing. This system is able to quantify the traditional commercial hairniness parameters, as $\mathrm{sH}(\%), \mathrm{CVH}(\%), \mathrm{H}$ and spectral analysis based on FFT. In addition several other parameters, novel in textile characterization, such as DRH (\%), IDRH (\%), UH $(\%)$ and spectral analysis based on FWHT and FDFI are readily available. The results obtained using this system to characterize a $4.20 \mathrm{~g} / \mathrm{Km}$ cotton yarn are presented.
\end{abstract}

\section{INTRODUCTION}

Y ARN hairiness is a key parameter characterizing yarn quality in the textile industry. Hairiness consists of small fibers that protrude from the main yarn core as shown in Fig. 1.

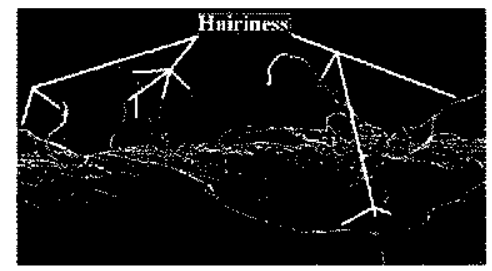

Figure 1. Electron microscope photograph of yarn hairiness (the small yarn fibers that protrude from the core)

For quantifying yarn hairiness two interconnected hardware setups are required: optical and electronic $[1,2]$.

Manuseript received January 15,2007 . This work was supported in part by the Portuguese Foundation (FCT) scholarship (BD/19028/2004)

V. II. Carvalho and F. O. Soares are with the Minho University, Dept Industrial Clectronics, Campus de $\Lambda$ zurèm, 4800-058 Guimarães, Portugal (phone: +351253510180 ; fax: +351253180189 ; c-mail: vearvalho@ dei.uminho.pt, fsoares $@$ dei.uminho.pt).

P. J. Cardoso and M.S. Belsley are with the Minho University, Dept, of Physics, Campus de Gualtar, 4710-057 Braga, Portugal (e-mail: pjcardosoafisica uminho pt, belsley (a) tisica.uminho pt)

R. M. Vasconcelos, is with the Minho University, Dept. Textile Engineering, Campus de Azurém, 4800-058 Guimarāes, Portugal (e-mail: rosa $(\bar{a}$ det.uminho.pt).

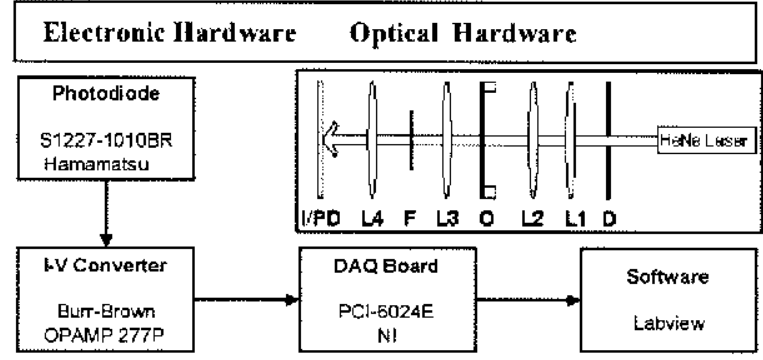

Figure 2. Custom developed electronic and optical hardware

The objective of the optical setup is to obtain a signal proportional to yarn hairiness in the final image plane (position of the photodiode in Fig. 2). Coherent light from $\mathrm{HeNe}$ laser is incident on a variable diaphragm to ensure a good transverse spatial profile. After the diaphragm, the laser beam passes through a two lens beam expander telescope (L1 and L2) and is directed to the yarn, placed in the object holder. The size of the object image is controlled by the lenses L.3 and L.4. A custom fabricated spatial filter (F) is placed in the Fourier plane of L3, to process the image, permitting only the high spatial frequencies in the image to propagate further (high pass spatial Fourier filter). This results in the contours of the edges of the yarn and associated hairs being highlighted while simultaneously eliminating the constant background. To establish a better SNR a linear polarizer (P) was placed before the object plane. Fig. 3 presents an example of an image obtained with the optical setup.

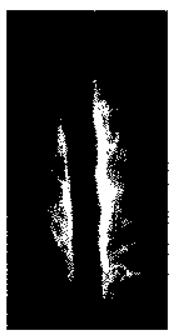

Figure 3. Example of an image obtained using the described optical setup

The objective of the electronic hardware is to obtain a voltage proportional to the brightness of the final image. The output of a trans-impedance amplifier connected to the photodiode is read by a Data Acquisition Board (DAQ). 
Previous studies have confirmed that when using this setup, the output voltage is proportional to the hairiness length [3].

Moreover, a statistical method to obtain the reference (without hairiness) was also developed. This method is based on the observation that when analyzing yarn using small sample lengths, an appreciable number of observations correspond to sections of yarn without hairiness (Fig. 4) [4].

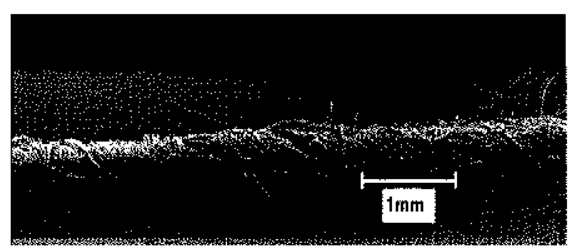

Figure 4. Electron microscope image showing that for this yarn, several sections of $1 \mathrm{~mm}$ are without hairiness

To obtain a viable reference value, the acquisition was performed in steps of $1 \mathrm{~mm}$ length, placing an aperture over the photodiode with an area of $1 \mathrm{~mm} \times 9 \mathrm{~mm}$.

\section{HAIRINESS STATISTICAL PARAMETERS}

This section presents the hairiness statistical parameters which are measured by the system we have developed. Standard parameters available using commercial equipments are: $\mathrm{H}, \mathrm{CVH}$ and $\mathrm{sH}$. However, our approach introduces several new ones, such as UH, DRH, IDRH and frequency diagrams of $\mathrm{H}$ variation. These parameters are measured considering the average hairiness as a yarn reference, as the contours of yarn are not considered hairiness. We now go on to briefly define these parameters.

\section{A. Hairiness Coefficient}

The hairiness coefficient $(\mathrm{H})$ corresponds to the length of hairiness per meter of yarn, as defined by equation 1 .

$H=\frac{l_{H}}{l}$

Where, $\quad 1_{\mathrm{H}}-$ total length of hairs (m) 1 - yarn length $(\mathrm{m})$

\section{B. Absolute mean deviation of hairiness coefficient}

The absolute mean deviation of the hairiness coefficient $\left(\mathrm{U}_{\mathrm{H}} \%\right)$ corresponds to the deviation of the hairiness from its average value. It is defined by equation 2 .
$U_{H}(\%)=\frac{100}{\bar{H} N} \sum_{i=1}^{N}\left|H_{i}-\bar{H}\right|$

Where, $\mathrm{H}_{\mathrm{i}}$ - current sample hairiness value

$\bar{H}$-average hairiness value during
evaluation time
$\mathrm{N}-$ number of samples

\section{Standard deviation of the hairiness coefficient}

The standard deviation of the hairiness coefficient is defined by equation 3 .

$s H(\%)=\sqrt{\frac{1}{N} \sum_{i=1}^{N}\left(H_{i}-\bar{H}\right)^{2}}$

\section{Coefficient of variation of the hairiness coefficient}

The coefficient of variation of the hairiness coefficient $\left(\mathrm{CV}_{\mathrm{H}}\right)$ is related to its standard deviation and its average value, as defined by equation 4 .

$C V_{H}(\%)=\frac{100}{\bar{H}} \sqrt{\frac{1}{N} \sum_{i=1}^{N}\left(H_{i}-\bar{H}\right)^{2}}$

\section{E. Deviation rate of hairiness coefficient}

The deviation rate parameter. DRH, characterizes the number of yarn samples with a hairiness that is outside given limits relative to the average yarn hairiness. To calculate the deviation rate, a function $p(\alpha, n)$ which assumes the value of ' 1 " if a sample is above or below these limits $(\alpha)$ and ' 0 ' if it is not, is defined by equation 5 [5]:

$P(\alpha, n)=\left\{\begin{array}{llc}1 & \text { if } & f(x) \geq \bar{H}(1+\alpha) \\ 0 & \text { if } & \bar{H}(1-\alpha)<f(x)<\bar{H}(1+\alpha) \\ 1 & \text { if } & f(x) \leq \bar{H}(1-\alpha)\end{array}\right.$

$\mathrm{DR}_{\mathrm{H}}(\%)$ as function of $\alpha$ is given by equation 6 :

$D R_{H \alpha}(\%)=\frac{\sum_{i=0}^{N-1} p(\alpha, n)}{N} 100$

The number of samples that exceeded the established limits for yarn hairiness is summed and divided by the total number of samples, yielding the deviation rate of hairiness result for a certain limit given by $\alpha$. 


\section{F. Integral deviation rate of hairiness coefficient}

The integral deviation rate parameter, IDR $_{\mathrm{H}}$, calculates the absolute mean deviation for several thresholds $(\alpha)$. This parameter is measured using the function, $y(n)$, shown in equation 7 [5]:

$y(n)=\left\{\begin{array}{ccc}|f(x)-\bar{H}(1+\alpha)| & \text { if } & f(x) \geq \bar{H}(1+\alpha) \\ 0 & \text { if } & \bar{I}(1-\alpha)<f(x)<\bar{H}(1+\alpha) \\ |f(x)-(\bar{H}-\alpha)| & \text { if } & f(x) \leq \bar{I}(1-\alpha)\end{array}\right.$

It is defined as a function of $\alpha$ in equation 8 :

$$
I D R_{H \alpha}(\%)=\frac{\sum_{i=0}^{N-1} y(n)}{\overline{H N}} 100
$$

According its definition, if $\alpha$ is zero, IDR $\mathrm{IH}_{\mathrm{H}}$ equals the value of $\mathrm{U}_{\mathrm{H}}$. So, with $\mathrm{IDR}_{\mathrm{H}}$ it is possible to quantify this characteristic $\left(\mathrm{U}_{\mathrm{H}}\right)$ not only for $0 \%$, but for values in the range of $[0$, max. variation $\%$ giving complete information of the hairiness deviation of the yarn at all limits.

\section{G. Frequency of hairiness coefficient}

The frequency diagrams calculate the histogram of hairiness coefficient variation, for certain predefined variation intervals in the range $[\mathrm{min}$. variation, max. variation] $\%[5]$.

\section{Signal Processing APPROACHES}

In the spectral analysis of yarn hairiness three different approaches had been used. The first is traditional FFT (Fast Fourier Transform), but considering only real values, to test the occurrence of sinusoidal patterns. The second is the FWHT (Fast Walsh Hadamard Transform) [5], which is computationally faster than the FFT, for detecting rectangular pattems. Lastly, the FDFI (Fast Impulse Frequency Determination) $[6,7]$ can be used to detect impulse patterns. The representation of FFT and FWHT are in terms of wavelength $(\lambda)$, using energy bands which integrate the harmonics within a certain interval, to reduce the overlapping influence on results. Moreover, this integration yields a result more readily understood by the yarn producer. Wavelengths are calculated considering the results in frequency as equation $9[5,7]$.

$$
\lambda(m)=l \frac{f_{a}}{1000 f_{d}}
$$

Where,

$$
\begin{aligned}
& \mathrm{l}=\text { width of sensor }(\mathrm{mm}) \\
& \mathrm{f}_{\mathrm{a}}=\text { acquisition frequency }(\mathrm{Hz}) \\
& \mathrm{f}_{\mathrm{d}}=\text { detected frequencies }(\mathrm{Hz})
\end{aligned}
$$

\section{RESLITS}

Using the described setup and custom developed software written using IabVIEW ${ }^{T M}$ [8] to acquire and process the data, some results of a test using a $4.2(\mathrm{~g} / \mathrm{Km}$ ) yarn (Fig. 5), are presented.

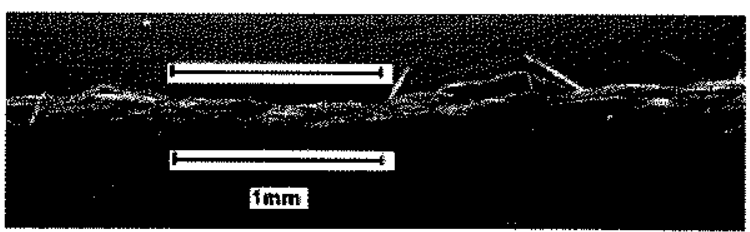

Figure 5. Electron microscope image showing that over the tested yarn, there is a section of $1 \mathrm{~mm}$ that is without hairiness

The experimental parameters are:

- Type of yarn: $100 \%$ combed cotton;

- Yarn linear mass: 4.2 tex ( $\mathrm{g} / \mathrm{Km}$ );

- Yarn diameter: 0.0037 tex $\wedge 0.5=0.08 \mathrm{~mm}$;

- Yarn speed $=6 \mathrm{~m} / \mathrm{min}$;

- Yarn length $=6 \mathrm{~m}$;

- Sample length = $1 \mathrm{~mm}$;

- Acquisition frequency $=100 \mathrm{~Hz}$;

- Signal without yarn $=0.12 \mathrm{~V}$;

- Hairiness length system sensitivity $=105.4$ $\mathrm{mV} / \mathrm{mm}$;

- Statistical Reference Value $=0.15 \mathrm{~V}$.

\section{A. Hairiness statistical parameter results}

Figure 6 presents the hairiness coefficient variation.

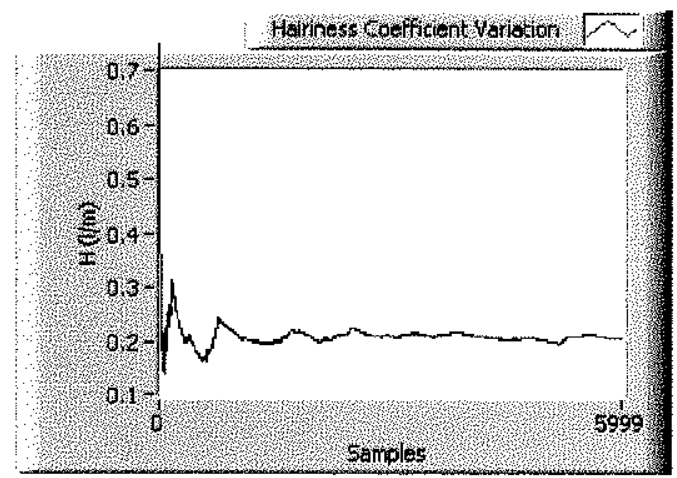

Figure 6. Diagram of the hairiness coefficient variation 
From the data presented in figure 6 we can conclude that this is a yarn with a rather value of hairiness. The values of $\mathrm{H}$ obtained are around $20 \mathrm{~cm}$ per metre of yarm.

Table I presents the results of $\mathrm{H}, \mathrm{CVH}(\%), \mathrm{sH}(\%)$ and $\mathrm{UH}$ $(\%)$.

TABLE I

MAIN STATISTICAL PARAMETERS RESUILTS OF H

\begin{tabular}{lc}
\hline Parameter & Result \\
\hline H & 0.21 \\
CVH (\%) & 10.48 \\
sH (\%) & 2.19 \\
IH (\%) & 4.43 \\
\hline
\end{tabular}

Analysing table I, a final value of 0.21 for $\mathrm{H}$ is obtained. It can also be stated that this value is relatively stable as proved by the low values of $\mathrm{CVH}(\%)$, sH $(\%)$ and $\mathrm{U}(\%)$ which are all inferior to $10.5 \%$.

Figure 7 present the results of Hairiness percentage variation relative to the average value.

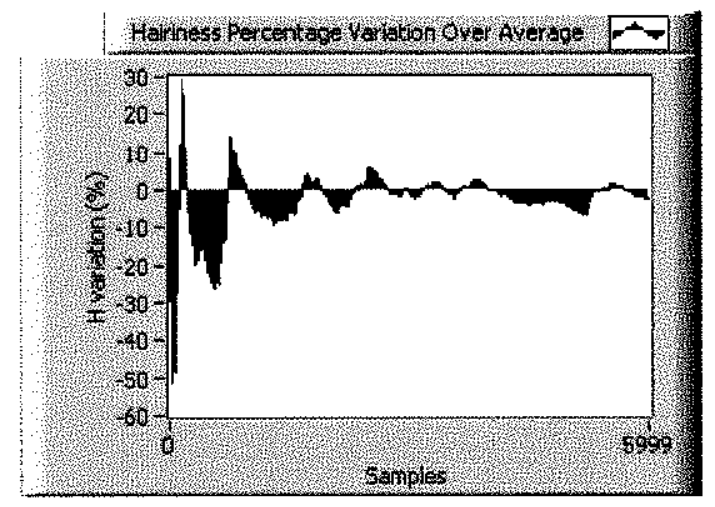

Figure 7. Diagram of hairiness percentage variation over average

Figure 7 confirms that the majority of the samples analyzed display a variation from the average, between $[-10,10] \%$, supporting the low statistical values of table $\mathrm{I}$.

Figures 8 and 9 present the results of deviation rate and its integral, respectively.

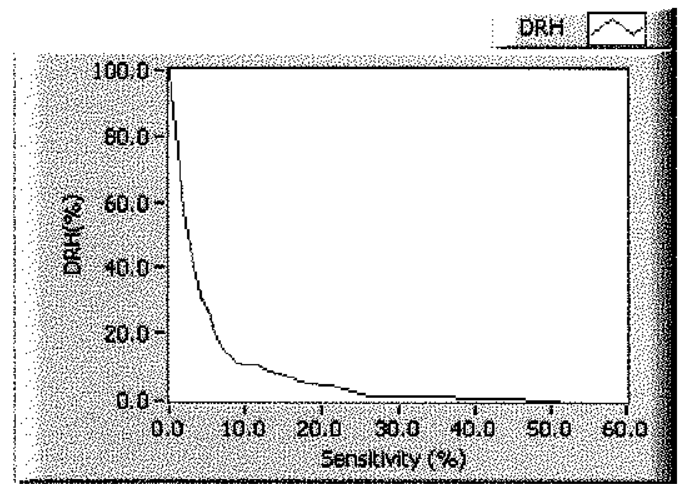

Figure 8. Diagram of hairiness deviation rate

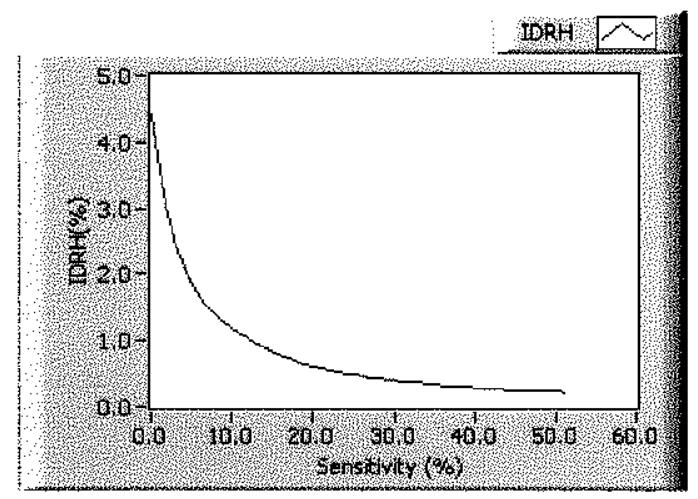

Figure 9. Diagram of hairiness integral deviation rate

As expected, the higher values are obtained at lower values of sensitivity and IDRH $(0 \%)=\mathrm{UH}(\%)$. It can be observed that the curves decrease rapidly; in the first $10 \%$ of sensitivity, the deviation rate values decrease from the maximum by around $80 \%$. Moreover, the range peaks are obtained between $[-50,30] \%$. These two characteristics are a consequence of the low level of hairiness, and its high stability.

Figure 10 presents the results of Hairiness Frequency Diagram.

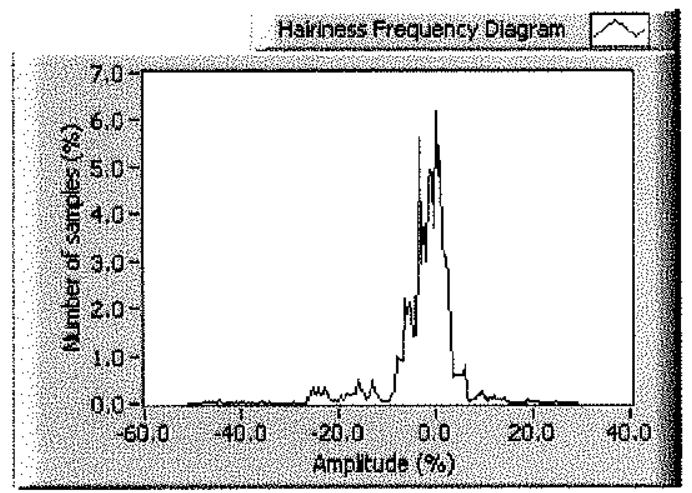

Figure 10. Diagram of hairiness frequency 
Analysing figure 10 , it can be seen that there is a high number of samples situated near the average $(0 \%)$, as should be expected from the fast decease observed in the results of the DRH and IDRH. Using this diagram, it is easy to obtain a distribution of the samples around the sensitivity steps.

\section{B. Signal Processing Results}

Figures 11 and 12 present the results of spectral analysis, using the real FFT and the FWHT, for the Hairiness percentage variation diagram (Fig. 7), respectively.

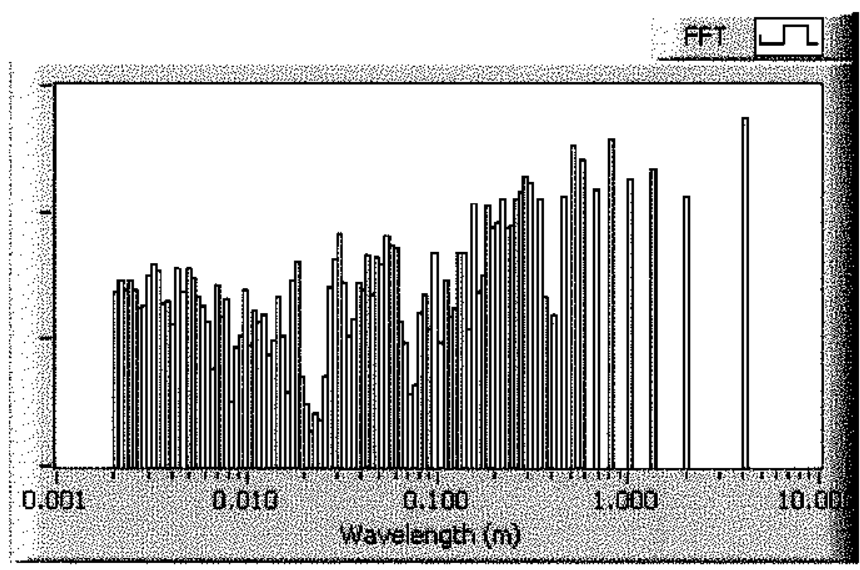

Figure 11. Diagram of hairiness spectral analysis based on FFT

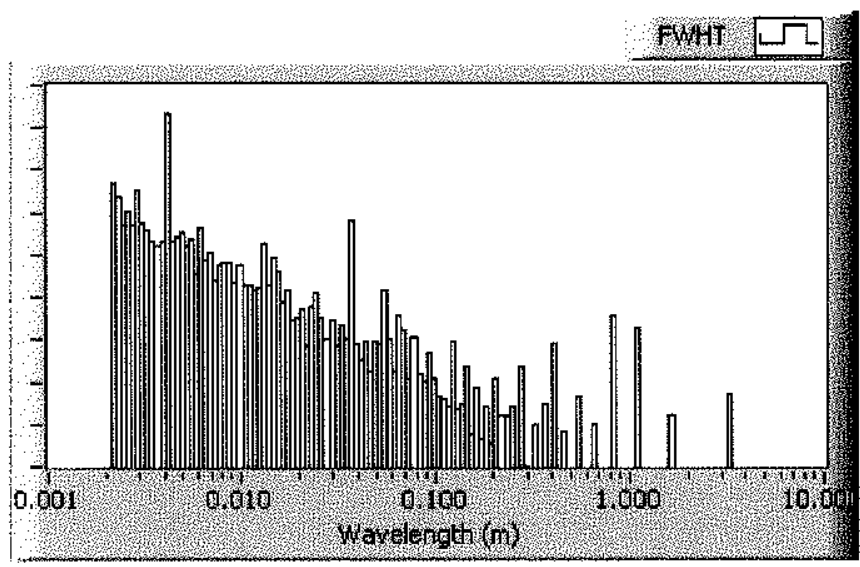

Figure 12. Diagram of hairiness spectral analysis based on FWHT

Analyzing figures 11 and 12 , it can be verified that generally both strategies detect errors in the same wavelength range, however with different levels. Figure 11 has main peaks around 2,3,10,11,40 cm and around 1 meter, but none of them are particularly dominating. This is probably due to the non- sinusoidal characteristic of the analyzed signal.
Figure 12 has main peaks around $5 \mathrm{~mm}, 3,40 \mathrm{~cm}$ and around I meter, which protrude significantly from the main tendency of the spectrum, probably to their pulse similarity with the analyzed signal. So, this technique is more adequate to analyze hairiness periodical variations. Moreover, in FWHT spectrum a power law is clearly identified, due to its linear scope over a logarithmic scale of wavelengths.

After the use of the previous signal processing strategies, the analysis of periodical impulse errors was also studied, for a threshold of $25 \%$. Figure 13 presents the error signal generated.

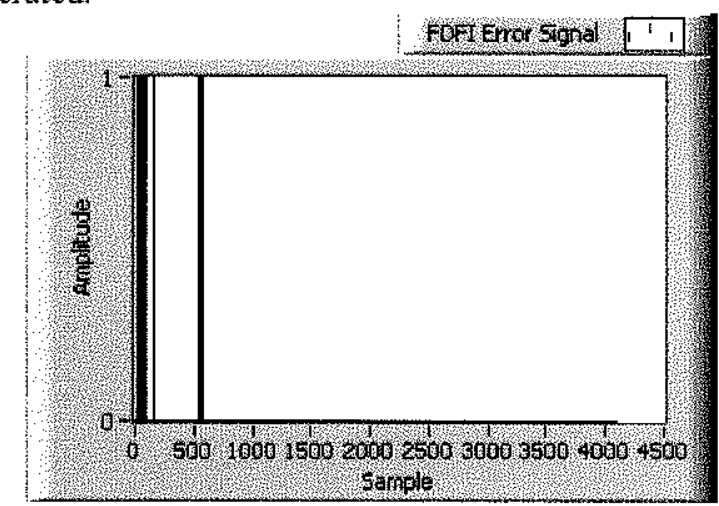

Figure 13. Diagram of FDFI error signal

In figure 13 the error signal occurs up to sample 600 in accordance to the threshold defined, because the source signal (fig. 7) has variations of $25 \%$ or greater only in the first 600 samples.

The error signal was subsequently tested with 1573376 periodical impulse errors, resulting from $((\mathrm{N} / 2+\mathrm{N} / 4+1) / 2 *(\mathrm{~N} / 2-\mathrm{N} / 4))[5,6]$ where $\mathrm{N}$ is the number of samples tested (4096).

The signal of row error belongs is presented in figure 14 .

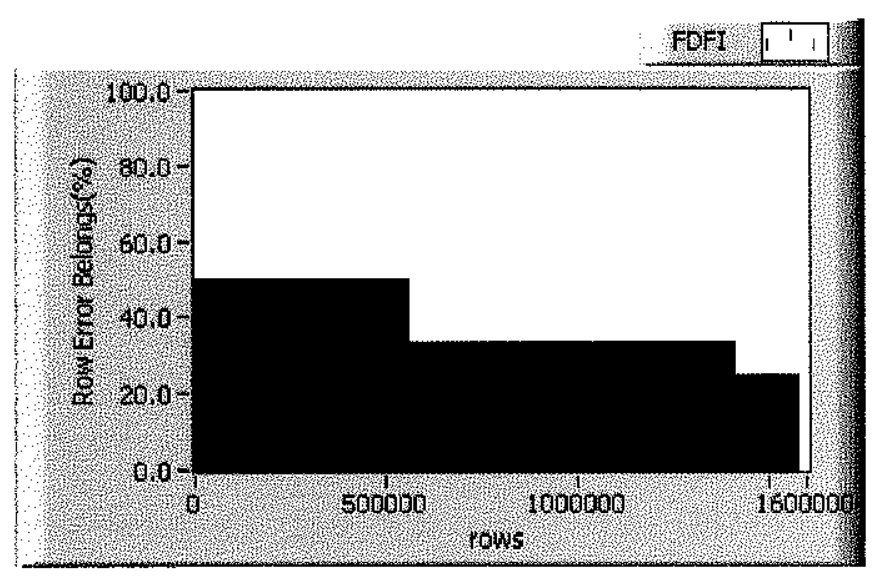

Figure 14. Diagram of FDFI row error belonging 
Figure 14 shows that there are not rows with an error belong of $100 \%$. This enables to the conclusion that for a threshold of $25 \%$, no periodical impulse errors are present in the analyzed signal.

\section{CONCLUSIONS AND FUTURE WORK}

At this point we are able to conclude that the methodology used (coherent optical signal processing plus electronics) yields reliable results. This is mainly the result of its low noise, stability and high linearity, factors which increase both resolution and accuracy.

Moreover, the statistical reference method previously developed enables the determination of the signal reference with the advantages of being non evasive and the possibility of being determined simultaneously with the data acquisition.

Using the statistical parameters $(\mathrm{H}, \mathrm{sH}, \mathrm{UH}, \mathrm{CVH}, \mathrm{DRH}$, IDRH and frequency diagrams) we could determine the level of hairiness, as well as its distribution and stability over the sample analyzed.

The introduction of the new statistical parameters, especially DRH and IDRH, are of utmost importance, as they present the number of samples / hairiness for different thresholds.

Considering the signal processing approaches, the real FFT and FWHT show, protruding peaks at the same wavelengths, however, in some cases with different amplitudes. This occurs because, the FFT is based on sinusoidal waves while the FWHT is based on rectangular waves. If the analyzed signal has variations, that are better approximated by sinusoidal waves it is expected to obtain larger amplitudes using the FFT approach (or vice-versa). However, FWHT is more adequate due to its significant protruding peaks form the main spectrum tendency. This analysis will be studied in the near future.

FDFI is an interesting strategy to determine impulse error patterns, as they are not detected by the previous approaches. The result obtained in a row error belong graph, can easily verify if a periodical impulse error, which is tested over all possibilities by specific matrix $[5,6]$, has occurred (row error belonging at $100 \%$ ).

This work is part of a R\&D project to develop an automatic system to characterize yarn quality, measure irregularities, hairiness and production yarn characteristics, which integrate both optical and capacitive sensors [9-11], as well as image processing techniques. With the extensive number of parameters that are potentially available, we expect to be able to significantly increase the information accessible to textile yarn manufacturers with a system that is both reliable and affordable.
We plan to adapt this system to simultaneously measure yarn diameter and correspondent mass variations as well as hairiness parameters by employing an appropriate photodiode linear array together with a low-pass spatial optical filter [12-15].

\section{ACKNOWI.FDGMENT}

The authors are grateful to the Portuguese Foundation (FCT) for funding through the scholarship (BD/ 19028/ 2004).

\section{REFERENCES}

11] V. Carvalho, P. Cardoso, M. Belslcy, R. Vasconcelos and F. Soares, "Determination of Yarn Hairiness IJsign (Optical Sensors", EUROSENSORS XX, 17-19 Septemher, Gothenturg, Sweden, 2006.

[2] V. Carvalho, P. Cardoso, M. Belsley, R. Vasconcelos and F. Soares, "Development of a Yarn Evenness Measurement and Hairiness Analysis System", IECON06, 7-10 November, Paris, France, 2006.

[3] V. Carvalho, P. Cardoso, M. Belsley, R. Vasconcelos and F. Soares, "Yarn Hairiness Parameterization Using a Coherent Signal Processing Tcchnique" (accepted for publication in Sensors and hctuators).

[4] V. Carvalho, P. Cardoso, M. Belsley, R. Vasconcelos and F. Soarcs, "A New Statistical Reference Method for Yarn Hairiness Quantification" (accepted for publication in ISIF, 2007).

[5] V. Carvalho, Parametrização de Fio Têxtil Baseada na Análise de Massa., Msc Thesis, Minho University, Guimarães, Portugal, 2002.

[6] J.L. Monteiro and C. Couto, "Pulse frequency calculation and estimation in yarn evenness analysis", IEEE Industrial Electronics Society, Orland, USA, 1995

[7] R. Furter, Evenness Testing in Yarn Production: par1 1, The Textile Institute and Zcllweger Uster AG, Manchester: 1982.

[8] url: www ni.com

[9] J.S. Ncves, A Irregularidade dos Fios Têxteis, sua origem, medição e análise, Oporto: 1968

[10] J.G.Pinto, Medição de Massa de Fio Textil, em Tempo Real, com $1 \mathrm{~mm}$ de Resoluçăo, Msc. Dissertation, Minho University, Guimarães: 2004.

[11] L. Baxter, Capacitive Sensors: Design and Applications, IEFE Press, NJ: 1997.

[12] J.W. Goodman, Introduction to Fourier Optics, McGraw-IIill, Greenwood Village, 1996.

[13] E.G. Steward, Fourier Optics: An Introduction, Dover Publications, New York, 2004

[14] P.M. Dutfieux, The Fourier Transform and its Applications to Optics 2nd Edifion, John Wilcy \& Sons, New York, 1983.

[15]C.K. Madsen, Optical Filter Design and Analysis: A Signal Processing Approach, Wiley-Interscience, New York: 1994 